\title{
The Perennial Use of the Green Fluorescent Protein Marker in a Live Vaccinia Virus Ankara Recombinant Platform Shows No Acute Adverse Effects in Mice
}

\author{
D. S. O. Daian e Silva ${ }^{1}$ - T. M. G. Pinho ${ }^{1}$ - M. A. Rachid $^{2}$ - D. F. Barbosa-Stancioli ${ }^{1}$ - F. G. Da Fonseca ${ }^{1}$
}

Received: 22 August 2018 / Accepted: 30 November 2018 / Published online: 15 March 2019

(C) Sociedade Brasileira de Microbiologia 2019

\begin{abstract}
Recombinant virus vectors represent a promising strategy for vaccine research. Among available viral vectors, members of the Poxviridae family — especially the modified Vaccinia virus Ankara (MVA) — stand out as immunogenic and safe vaccine platforms. Because MVA usually does not produce plaques in cell culture, visible selection markers such as the green fluorescent protein (GFP) are frequently incorporated into the constructions in order to facilitate the recognition of recombinants. However, these genetic markers have to be removed before any clinical trial. Here, we evaluated the acute responses generated in mice immunized with a MVA vector in which the GFP marker was not removed. We observed no differences in neutrophil, monocyte, or total leucocyte recruitment among animals inoculated with MVA or MVA-GFP. Likewise, there were no differences in neutrophil activation between mice groups. Hepatic functions were not altered in either MVA or MVA-GFP-inoculated mice, and we observed no histopathological alterations in different tissues from virus-inoculated animals. In conclusion, the presence of GFP is innocuous to immunized animals and do not alter acute physiopathological responses to the MVA vector. We suggest that keeping the GFP marker may be a good strategy for vaccine development, production, and evaluation.
\end{abstract}

Keywords MVA-based vaccine $\cdot$ Green fluorescent protein $\cdot$ GFP $\cdot$ Acute responses

$\begin{array}{ll}\text { Abbreviations } \\ \text { ALT } & \text { Alanine aminotransferase } \\ \text { AST } & \text { Aspartate aminotransferase } \\ \text { CEFs } & \text { Chicken embryo fibroblast cells } \\ \text { GFP } & \text { Green fluorescente protein } \\ \text { MPO } & \text { Myeloperoxidase } \\ \text { MVA } & \text { Wild-type modified Vaccinia virus Ankara } \\ \text { MVA-GFP } & \text { Modified Vaccinia virus Ankara-expressing } \\ & \text { GFP protein } \\ \text { MOI } & \text { Multiplicity of infection } \\ \text { PFU } & \text { Plaque forming unit } \\ \text { VACV } & \text { Vaccinia virus }\end{array}$

Responsible Editor: Mauricio Nogueira

F. G. Da Fonseca

fdafonseca@icb.ufmg.br

1 Laboratory of Basic and Applied Virology, Departmento de Microbiologia, Instituto de Ciências Biológicas, Universidade Federal de Minas Gerais, Belo Horizonte, MG, Brazil

2 Departamento de Patologia, Instituto de Ciências Biológicas, Universidade Federal de Minas Gerais, Belo Horizonte, MG, Brazil

\section{Introduction}

Vaccination is an iconic approach to the management of infectious diseases. The power of vaccination as a cost-effective medical intervention can be exemplified by the vaccinemediated eradication of important human and animal infectious diseases, such as smallpox and rinderpest, respectively. Despite past and present medical achievements, the continuous emergence and reemergence of pathogens - causing from local outbreaks to global epidemics - are a real threat. Climate changes, uncontrolled urbanization and globalization - all hallmarks of modern society - are some of the main facilitators of disease transmission and the buildup of an epidemic. Moreover, the widespread development of pathogen resistance to antibiotics and antivirals has made it increasingly difficult to treat dozens of important infectious diseases, human and veterinary. In such pragmatic scenario, vaccination is likely to remain as one of the very fundamental pillars of public health [1].

Although vaccines are indisputably critical as a general way to deal with infectious diseases, vaccine-preventable diseases are largely outnumbered by infections to which no current vaccine solution exists — or are only partially effective [2, 
3]. The reasons for such gap are many, and range from unattractive revenues from the pharmaceutical industry viewpoint to the technical difficulties seen in the quest for efficient immunogens against several infections such as dengue, hepatitis $\mathrm{C}, \mathrm{HIV}$, and others. Concerning the last aspect, it has become increasingly consensual that if a vaccine against a given infectious disease is still nonexistent today, it is likely that classical approaches to vaccine development have failed to deliver appropriate levels of protection against such infection [4]. On the other hand, a plethora of nonclassic approaches to vaccinesfrom viral vectors to nanostructured immunogens - have been developed and tested lately, many with significant success.

Poxviruses have been inherently linked to the development of virology and modern vaccinology. Vaccinia virus (VACV), the prototypic member of the Poxviridae family, was the first animal virus to be microscopically visualized, purified, accurately titrated, chemically characterized, and grown in cultured cells [5]. Smallpox - caused by Variola virus, also a poxvirus - is considered by many as the most deadly infectious disease in the history of mankind. The disease was the first (and only, until today) human infection to be eradicated by means of vaccination, and it was done so through the use of a live vaccine consisting of VACV, which is antigenically and genetically related to Variola virus. After the eradication of smallpox, interest on poxvirus-derived vaccines veered towards their use as live recombinant vectors, especially due to the identification of more attenuated VACV strains such as $\mathrm{LC} 16 \mathrm{~m} 8$ (a Vaccinia virus Lister clone) and modified Vaccinia virus Ankara (MVA) [6, 7].

MVA was developed through a process of attenuation in which the parental VACV - the Ankara strain - was subjected to serial passages in primary chicken embryo cells. The resultant mutant strain presented a phenotype of low virulence as well as the inability to productively multiply in most mammalian cells whilst retaining its immunogenicity, allowing it to be used as a safe and efficient vaccine against smallpox [8]. The eradication of smallpox coincides historically with the first descriptions of poxvirus-based expression vectors [9], which were quickly followed by the popularization of MVA as a recombinant vaccine vector against many infectious diseases, most of them in the veterinary field [7]. Due to its significant lack of virulence, MVA causes very little cytopathic effects even in susceptible cells. Although this is a good feature in terms of attesting the virus attenuation and consequent safety, it complicates practical matters such as the selection and monitoring of recombinant clones. To circumvent this, the development of MVA-based vectors usually includes the incorporation of genes coding for selectable markers into the recombinant's genome. One popular marker is the gene coding for the green fluorescent protein (GFP), from the algae Aequorea victoria, which is able to emit a bright green fluorescent light when stimulated with ultraviolet (UV) light [10].
Although useful in the initial construction steps of a MVAbased vector, it is common sense that the selectable gene marker must be removed before clinical trials using the vector can be carried out. Nonetheless, the removal of this visual aid from the vaccine vector adds further steps to the development of the intended vaccine and makes future analysis of the vaccine more laborious, including processes such as titrations, quality control, and stability assessments. As a consequence, all this add up to the final cost of the vaccine development. Thus, keeping in mind the burden of removing GFP from the final construct, we asked whether removal of GFP selectable marker would be really necessary in the context of the acute use of a MVA-based vaccine vector.

\section{Material and methods}

\section{Production and maintenance of the cells}

The MVA vector expressing the GFP protein (MVA-GFP) was selected, amplified, and titrated in chicken embryo fibroblasts (CEFs). To obtain the cells, embryonated eggs with 10 days of laying were used. The embryos were prepared by removing limbs and major internal organs; cells were trypsinized with $0.25 \%$ trypsin- $0.02 \%$ EDTA (Sigma-Aldrich, USA) at $37{ }^{\circ} \mathrm{C}$ for 15 min under agitation and the final suspension was slowly filtered. Trypsin activity was stopped with the addition of $200 \mathrm{~mL}$ Dulbecco's modified Eagle's medium (DMEM) (Sigma-Aldrich, USA) supplemented with 7.5\% NaHCO3, antibiotics $(100 \mu \mathrm{g} / \mathrm{mL}$ streptomycin and $100 \mathrm{U} / \mathrm{mL}$ penicillin), antifungal (fungizone at $25 \mu \mathrm{g} / \mathrm{ml}$ ), and $10 \%$ fetal bovine serum (Gibco, USA), and after centrifugation the cells were suspended in the same medium. Cells were incubated at $37^{\circ} \mathrm{C}$ with $5 \% \mathrm{CO}_{2}$ and showed confluence in approximately 2 days, being sub-cultured at every 3 days.

\section{Construction of MVA-GFP}

In order to evaluate the acute impact of GFP inoculated in vivo through the use of a viral vector, we constructed a GFPexpressing MVA. The parental virus was kindly provided by Dr. Bernard Moss (National Institute of Health-NIAID/NIH, Bethesda, USA) on its 554th passage. CEF monolayers were infected with MVA at $0.01 \mathrm{MOI}$ and transfected with the pLW44 transfer plasmid [11], which contains a cDNA encoding the GFP protein under a strong VACV late promoter. The transfection solutions were prepared during the viral adsorption using the Lipofectamine 2000 reagent (Invitrogen, USA), pLW44-GFP plasmid, and Optimem serum-free medium (Gibco, USA). The transfection solution was added to wells containing infected CEFs and cells were incubated for $5 \mathrm{~h} / 37{ }^{\circ} \mathrm{C}$. After this period, the transfection solution was removed, replaced with DMEM medium, and the plates were 
incubated for further 48 h. Finally, cells were harvested, pelleted, and disrupted by freeze/thawing. The MVA-GFP recombinants were visually identified on the basis of GFP expression, plaque-purified, and selected though consecutive passages in CEFs. The recombinant clone was subsequently amplified by successive passages in culture plates and flasks containing CEFs. Virus purification was performed in $36 \%$ $(w / v)$ sucrose cushion. Virus stocks were titrated in CEFs and frozen at $-80{ }^{\circ} \mathrm{C}$ until use.

\section{Mice and infections}

Eight- to 12-week-old Balb/C female mice were inoculated with either MVA-GFP or wild-type MVA. Animals were divided in five groups of five animals each, as follows: (i) control (animals that received only phosphate buffered salinePBS 1-); (ii) animals inoculated with MVA-GFP and euthanized $24 \mathrm{~h}$ after infection; (iii) animals inoculated with MVAGFP and euthanized $72 \mathrm{~h}$ after infection; (iv) animals inoculated with the parental MVA and euthanized $24 \mathrm{~h}$ after infection; and (v) animals inoculated with the parental MVA and euthanized $72 \mathrm{~h}$ after infection. Mice were anesthetized intraperitoneally with a solution of ketamine/xilazine in PBS and inoculation was performed with $10^{8}$ pfu of virus through injection on the base of the tail vein. Animals were euthanized at 24 and $72 \mathrm{~h}$ after immunization and had blood, spleen, liver, and lungs collected for processing. Blood was collected via the brachial plexus and serum was obtained. All procedures reported here are in accordance with the ethical principles of animal experimentation adopted by the Ethics Committee for Animal experiments from Universidade Federal de Minas Gerais (CETEA/UFMG—protocol 273/2008).

\section{Myeloperoxidase quantification}

The accumulation of neutrophils into pulmonary tissue, indicative of an inflammatory process, was inferred by assaying the myeloperoxidase activity (MPO), as described [12, 13]. Briefly, lung fragments were homogenized in appropriate buffer $\left(0.1 \mathrm{M} \mathrm{NaCl}, 0.02 \mathrm{M} \mathrm{NaPO}_{4}, 0.015 \mathrm{M}\right.$ NaEDTA; $\mathrm{pH} 4.7$ ) (1 $\mathrm{g}$ to $19 \mathrm{ml}$ of buffer) and centrifuged at $9279 \times \mathrm{g}$ for $10 \mathrm{~min}$. The precipitate was subjected to hypotonic lysis (15 $\mathrm{mL}$ of $0.2 \% \mathrm{NaCl}$ for $30 \mathrm{~s}$ followed by the addition of an equal volume of solution containing $1.6 \% \mathrm{NaCl}$ and $5 \%$ glucose) and centrifuged. The new precipitate was suspended in 0. $05 \mathrm{M} \mathrm{NaPO}_{4}$ buffer ( $\mathrm{pH}$ 5.4) containing $0.5 \%$ of hexadecyltrimethylammonium bromide (HTAB) and homogenized. Aliquots of the suspension underwent three cycles of freezing and thawing in liquid nitrogen and were successively centrifuged for $15 \mathrm{~min}$ at $9279 \times \mathrm{g}$. The final supernatant was utilized for the enzymatic assay. Inferred MPO activity was measured on the basis of changes in optical density (OD) at $450 \mathrm{~nm}$ using tetramethylbenzidine $(1.6 \mathrm{mM})$ and $\mathrm{H}_{2} \mathrm{O}_{2}$
(0.5 mM). Results were expressed in relative units, based on a standard curve of numbers of neutrophils versus the OD. Mice peritoneal neutrophils were processed in the same way. The results were plotted using GraphPad Prism 4 (GraphPad Inc., San Diego, CA, USA). The data distribution was tested by Kruskal-Wallis and one-way ANOVA tests, followed by Dunn's multiple comparisons' test whenever pertinent. Values of $p<0.05$ were considered statistically significant.

\section{Liver enzymes' quantification: Aspartate aminotransferase (AST) and alanine aminotransferase (ALT)}

Serum samples collected from BALB/C mice infected either with MVA or MVA-GFP were submitted to a colorimetric assay to quantify the liver enzymes AST and ALT. For this, a Bioclin kit (Brazil) was used according to the manufacturer's instructions. The generated results were analyzed using GraphPad Prism 4 and one-way ANOVA. The NewmanKeuls multiple comparison's test was used to analyze the variance between groups. Values of $p<0.05$ were considered statistically significant.

\section{Leukocyte count}

\section{Total cell count}

Leukocytes from blood samples of infected mice were counted in a Neubauer chamber. Cell assessment was performed with the aid of the Turk dye (gentian violet solution and acetic acid).

\section{Differential cell counts}

Blood smears on glass slides were stained using the Quick panoptic kit (Laborclin, Brazil) according to the manufacturer's instructions. Collected data were analyzed using GraphPad Prism 4 and one-way ANOVA tests. The Newman-Keuls multiple comparisons test was used to analyze variance between groups. Values of $p<0.05$ were considered statistically significant.

\section{Histopathology}

Animals had sections of the liver, spleen, and lungs removed for histopathological analysis in order to evaluate possible tissue damage caused by the inoculation of MVA or MVAGFP. The samples were fixed and stained according to standard histological procedures for hematoxylin eosin (H\&E) staining and were examined under light microscopy. 


\section{Results}

\section{The green fluorescent protein as a viral selection marker}

The MVA-GFP selection was done with the aid of the GFP protein expression as the distinction between MVA-infected and uninfected cells under transmitted light microscopy is quite difficult if no visual aid is utilized. Likewise, distinction between cells containing recombinant or parental viruses is also difficult - if not impossible - when no selection marker is employed in the recombinant construct. Cells infected by the GFP-expressing MVA vector (Fig. 1a) are readily distinguishable from either uninfected cells or cells infected with the parental MVA (not shown). As shown in Fig. 1b, the very same foci shown on Fig. 1a reveals very little cytopathic effects when analyzed under regular transmitted light microscopy, making it very difficult to pinpoint cells infected with the MVA vector. Although some cell rounding and vacuolization may suggest virus infection, the absence of a visual aid to identify cells infected by recombinant MVAs definitely complicates the identification and selection of recombinant viral clones as MVA causes no readily visible plaques in cell monolayers. Obviously, the detection of intracellular GFP requires irradiation of near UV or blue light; therefore, a microscope with fluorescent capabilities is necessary for virus screening and monitoring. However, different from serological and most genetic markers, no dye, antibiotic, substrate, or any other screening agent have to be added to the monolayer in order to select recombinant viruses, which reduce costs and time during their construction. Indeed, due to its plasticity in many biological settings, GFP has been widely used as a molecular marker to monitor protein localization patterns, gene expression, intracellular protein traffic in living cells, and many other applications [14].

\section{Inflammation evaluation in MVA or MVA-GFP infected cells}

\section{MPO activity}

We chose the lungs as a target organ to be analyzed because MVA and other VACV strains present intense tropism to lung tissues in mice, even when not inoculated through the intranasal route [15]. In fact, virus inoculation through the tail vein will carry virus particles straight to the lungs after going through the heart, leading to the infection of pulmonary tissue. In order to infer the occurrence of inflammation after viral infection, lung fragments of mice inoculated either with MVA or with MVA-GFP were analyzed through the measurement of MPO activity as an indirect way to look for the accumulation of neutrophils in these tissues (Fig. 2). We observed no statistically significant differences in MPO activity between any of the groups - including the unimmunized controls - in both 24 or $72 \mathrm{~h}$ post-immunization (Fig. 2) (Kruskal-Wallis oneway ANOVA $p>0.05$, Dunn's multiple comparison test with $p>0.05$ ). The MPO enzymatic activity is an indirect marker of activation, degradation, or infiltration of neutrophils in inflamed tissues [16], and several inflammatory stimuli, acute and chronic, can result in MPO secretion into the bloodstream [17]. Although produced by other phagocytes as a key regulator of the respiratory burst during phagocytosis [18], MPO is more importantly associated with neutrophils activity during the early stages of an inflammatory reaction [19]. The expression of GFP by the MVA vector, compared to the virus that does not express the fluorescent marker, seems to have not altered significantly the neutrophil activation upon infection (Fig. 2).

\section{Neutrophils and monocytes recruitment}

Because no statistically supported differences were detected between virus-infected animals and the mock-infected controls, concerning MPO activity, we asked whether neutrophils would have been recruited at all during infections. Neutrophil recruitment was confirmed by the direct cell count using a Quick panoptic kit, as described. We observed no statistical differences in neutrophil counts among infected animals, either at 24 or $72 \mathrm{~h}$ post-inoculation, regardless of the GFP presence (Fig. 3-neutrophils). Likewise, there were no differences between the immunized groups, at 24 or $72 \mathrm{hpi}$, when monocytes were evaluated (Fig. 3-monocytes). On the other hand, neutrophil and monocyte counts in placebo-inoculated animals were statistically different from counts in infected
Fig. 1 Cells inoculated with MVA-GFP virus. Chicken embryo fibroblasts were inoculated with the recombinant MVA expressing GFP and observed under ultraviolet light (a) or transmitted light (b). Magnification: $\times 400$
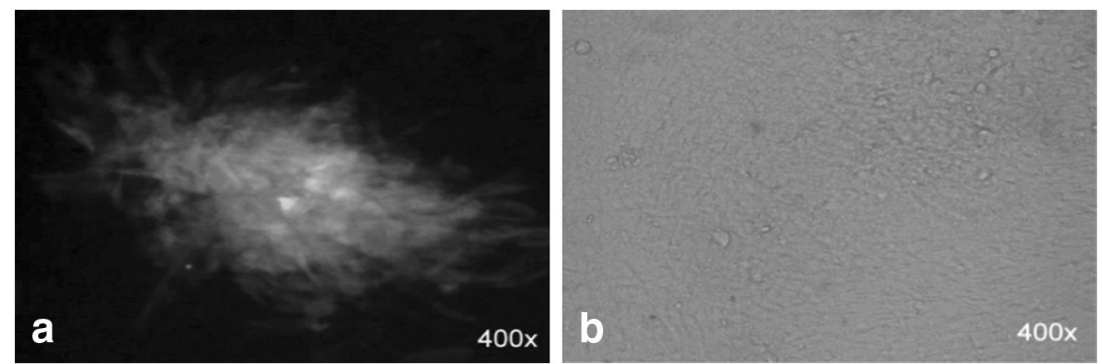
Fig. 2 Quantification of myeloperoxidase enzyme activity in the lungs of MVA-infected and non-infected mice. MPO activity in lung samples from mockinfected mice (placebo, PBS), and animals inoculated with MVA or MVA-GFP, was evaluated at 24 and $72 \mathrm{~h}$ post-infection. MPO activity is given in relative units. No statistical difference between the groups analyzed was observed. Samples were tested by KruskalWallis one-way ANOVA and Dunn's multiple comparisons' test
MPO

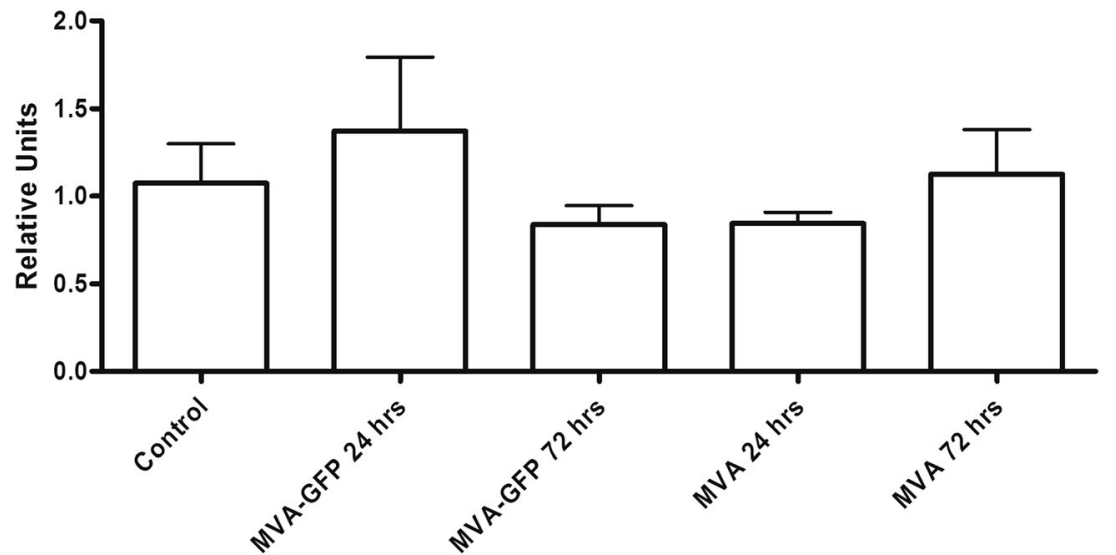

animals, independently of GFP expression. Neutrophil counts in mock-infected mice were significantly lower than those seen in MVA-infected animals, as expected, and the difference can be attributed to the activation of innate immune responses triggered by the MVA inoculation. Conversely, monocyte counts in placebo-treated mice were significantly higher than counts in MVAinoculated animals, regardless of GFP production. There was no difference in total leucocyte counts for all analyzed groups (Fig. 4).

A plausible explanation for the differences between MPO activity and neutrophil recruitment in infected animals is that neutrophils would be only being recruited and not activated, thus precluding the release of MPO and consequently its detection. Another possible explanation would be that the MPO enzyme may have been inactivated or even eliminated from the analyzed tissue through binding to macrophage mannose receptors [20].

\section{Analysis of the hepatotoxicity in infected mice}

At last, we looked at the liver conditions in animals infected with MVA vectors in order to check if the differential expression of GFP would induce liver alterations. In fact, the liver can be injured during the onset of many diseases that are systemic or that predominantly affect other organs. Therefore, it can be used as an indicator of health status and pathogenesis processes in a number of etiological scenarios [21]. Likewise, the systemic quantitation of liver enzymes is a generally accepted way to assess liver function and damage $[22,23]$; thus, we evaluated levels of aspartate aminotransferase (AST) and alanine aminotransferase (ALT) in the
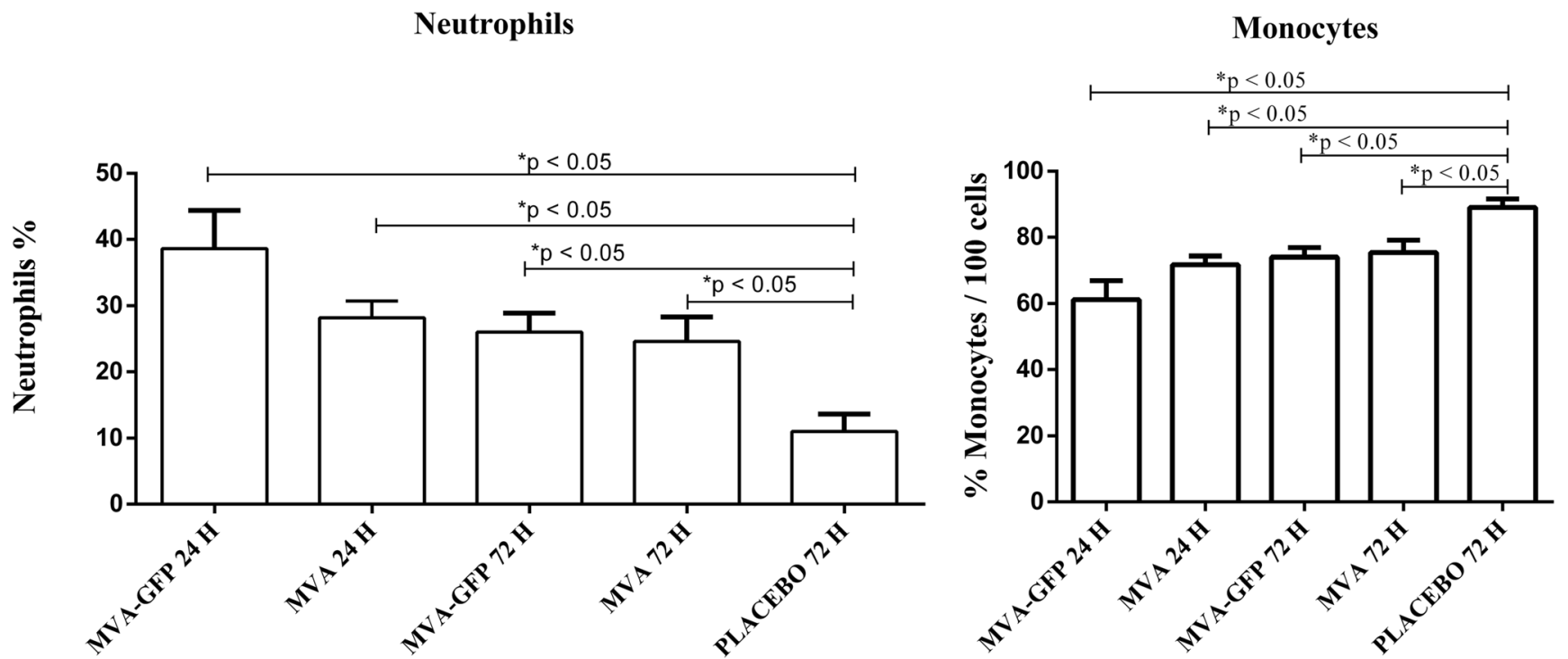

Fig. 3 Quantification of neutrophils and monocytes in the blood of MVAinfected and non-infected mice. Differential counts of neutrophils and monocytes in blood samples from mock-infected mice (placebo, PBS)

and animals inoculated with MVA or MVA-GFP were carried out at 24 and $72 \mathrm{~h}$ after infection. The Newman-Keuls test was used to analyze the variance between the groups 
Total leukocyte count

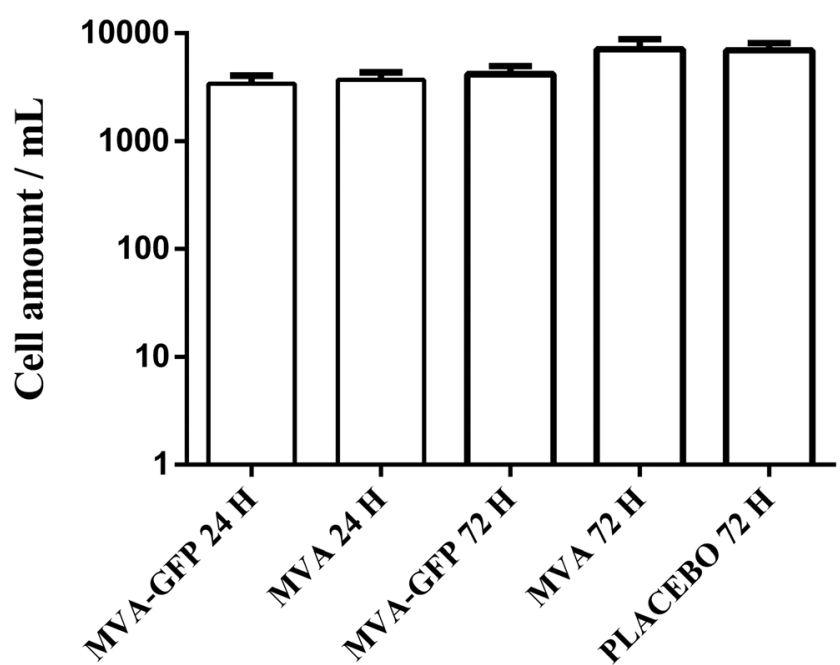

Fig. 4 Quantification of total leukocytes in the blood of MVA-infected and non-infected mice. Total leukocyte count in blood samples from mock-infected mice (placebo, PBS) and animals inoculated with MVA or MVA-GFP were carried out at 24 and $72 \mathrm{~h}$ after infection. The Newman-Keuls test was used to analyze the variance between the groups

bloodstream of animals inoculated with MVA vectors expressing or not GFP. Serum samples collected from BALB/C mice infected with MVA or MVA-GFP were submitted to colorimetric assays for ALT and AST detection. Non-infected animals were also evaluated as controls. We found no evidence of hepatotoxicity related to the MVA vector, independently of GFP expression (Fig. 5).

The enzymes AST and ALT are able to metabolize glutamate in the peripheral blood, leading to a decrease in the circulating levels of this amino acid [22]. Such enzymes are markers of liver toxicity caused by inflammatory processes or the use of medication, and their measurement is an efficient way to distinguish liver damage from that of other organs [23]. The ALT activity in the liver is about 3000 times higher than in serum; however, in the case of hepatocellular damage or death, it is released and the enzyme concentration in serum increases considerably. Therefore, the fact that AST and ALT levels were undistinguishable in the plasma from MVAinfected animals in relation to non-infected controls, corroborate both the liver-innocuity of MVA as a safe vector and the absence of liver toxicity caused by GFP expression by the viral vector.

\section{Histopathological characterization of MVA-GFP}

To further look for possible toxicity effects caused by GFP production into the liver of animals inoculated with MVAGFP, we analyzed sections of hepatic tissue from MVAGFP-infected mice and compared with images from the wild-type MVA-infected controls. No significant
ALT
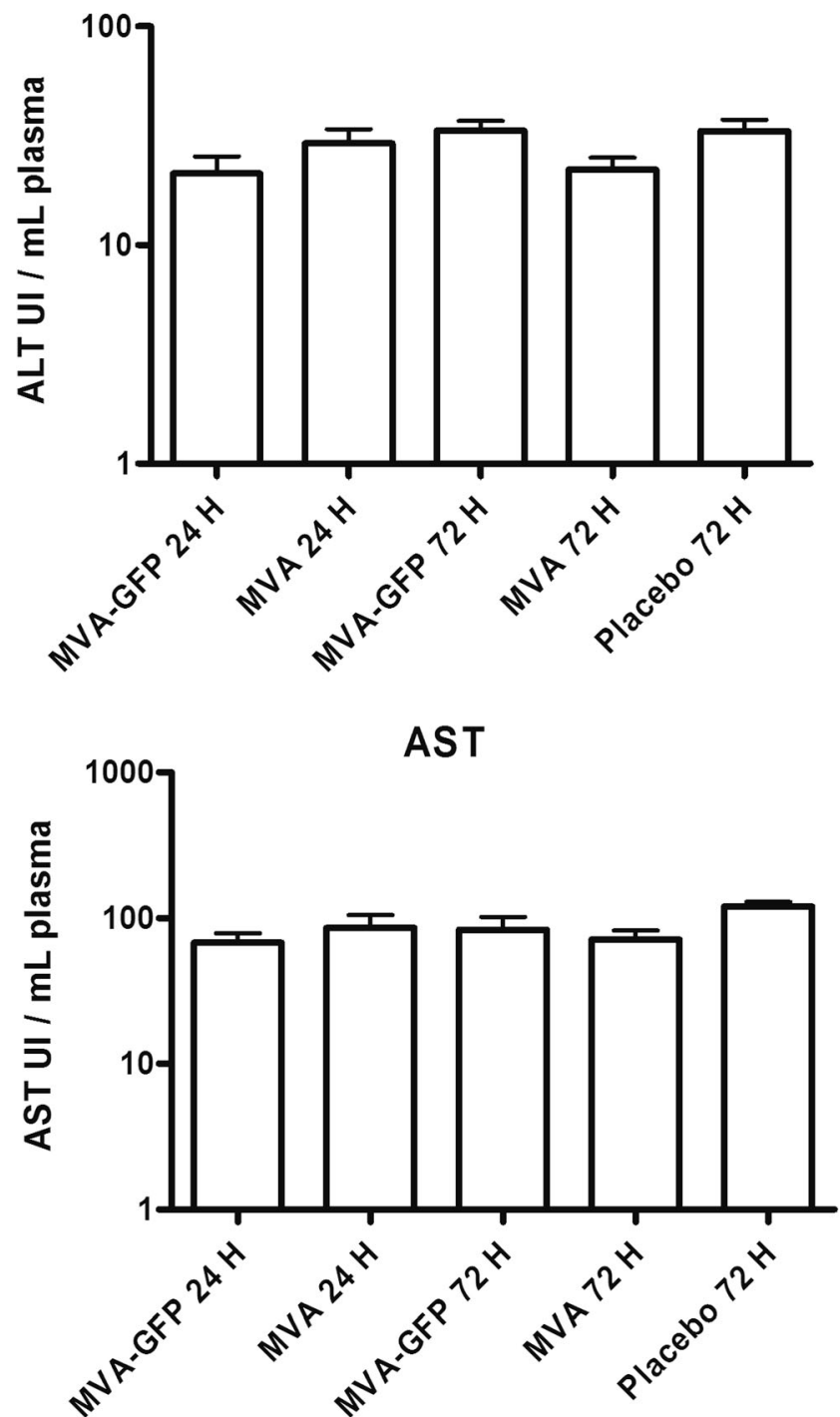

Fig. 5 Quantification of hepatic enzymes' activity in the sera of MVAinfected and non-infected mice. ALT and AST activities in the sera of mock-infected mice (placebo, PBS), and animals inoculated with MVA or MVA-GFP, were evaluated at 24 and $72 \mathrm{~h}$ post-infection. No statistical difference between the groups analyzed was observed. Samples were tested by Kruskal-Wallis one-way ANOVA and Dunn's multiple comparisons' test

histopathological abnormalities were observed in the liver of animals either in the presence or in the absence of GFP (Fig. 6), corroborating the AST and ALT findings. We extended the histological evaluations to the lungs - primary site of virus infection - and to the spleen, as vaccinia virusesincluding the MVA strain - present significant tropism to the spleen upon mouse infection [15, 24]. Sections of both tissues revealed no particular signs of pathology or cytotoxicity (Fig. 6), either in the presence or absence of GFP expression, similarly to what was found in the liver. Indeed, Ramirez and colleagues had shown that intranasal inoculation of MVA into mice caused no signs of edema, absence of cell infiltration 
Fig. 6 Histological aspects in MVA-infected organs. Thin sections from the liver $(\mathbf{a}-\mathbf{b})$, spleen (c- $\mathbf{d})$, and lung (e-f) of mice infected with wild-type MVA of MVA-GFP were prepared and $\mathrm{f}$ H\&E-stained. Magnification: ad: $\times 200$, e-f: $\times 400$. Figures are representative of many analyzed fields for each sample
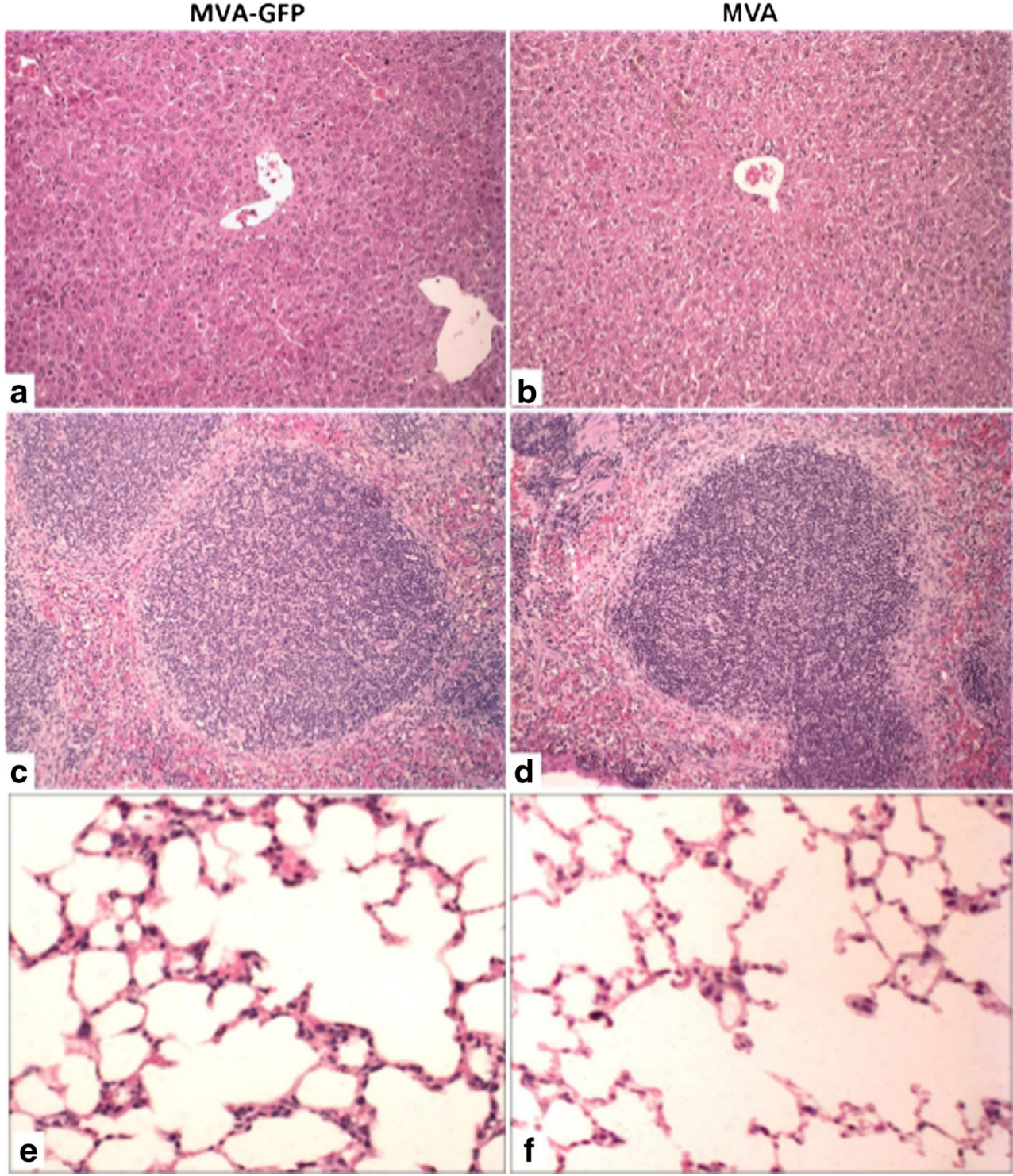

around bronchial tissues, and no accumulation of cell debris [15]. Even when expressing recombinant antigens, when immune responses to the transgenic antigen may intensify inflammation, signs of histopathological damage caused by the MVA vector is practically nonexistent [25, 26]. According to the results presented here, the perennial expression of GFP as part of the MVA vector seem to cause no alterations on the safety and innocuity of this VACV strain upon immunization/inoculation into the target host.

\section{Discussion}

As noted by Plotkin and colleagues [3], the development of vaccines against important infectious diseases have been hampered by three main factors: the complexity of some of the targeted diseases, which have challenged scientists for years and consumed enormous amounts of resources with no palpable positive outcomes; the diminishing number of vaccine manufacturers willing to devote human and financial resources to vaccine development; and the prevailing business model in which vaccines with large potential markets are prioritized over costly vaccine researches on less potentially lucrative immunogens. In the first case, new approaches to vaccine development have renewed hopes that the vaccine development against iconic infectious diseases with no previous vaccine solution may be at hand. Viral vectors based in poxvirus, adenoviruses, and other viral and bacterial backbones are among the new strategies. Nonetheless, such cutting-edge approaches may implicate in increasing costs for vaccine development and production, which may lead straight to the second and third aforementioned reasons for vaccine research 
curtails. Pragmatically, reduced costs are a necessary feature if any vaccine research and development agenda is to be implemented. Because many viral vectors are attenuated, they need a genetic marker - such as GFP - as an aid to select recombinant clones. These markers are later on removed before clinical trials. On the other hand, what if some of these markers are not removed? This could shorten steps of development and, consequently, reduce costs. In this case, could they induce toxicity to immunized subjects? We evaluated the possible negative effects in an acute mice model using recombinant MVA inoculation. According to our results, we detected no acute systemic or inflammatory adverse effects in relation to the expression of GFP. Nonetheless, a discrepancy in the placebo groups was observed when MPO activity and neutrophil counts were analyzed and compared. We found no statistically supported differences between measured MPO activities in mock-infected or MVA-infected cells, regardless of GFP expression. However, although no differences were found in infected animals concerning the presence of neutrophils in the lung, placebo-inoculated animals showed less neutrophils, as expected. If there was less neutrophils in the lung of uninfected animals, why would the MPO activity be equal among all groups? MVA is known to attract neutrophils the site of infection in a process that is dependent on the expression of C$\mathrm{C}$ chemokines and complement components [27, 28]; nevertheless, the stance of neutrophil degranulation and MPO release during infection with this virus is currently not clear and remains to be determined.

Obviously, retaining such genetic marker in a vaccine platform may give rise to many other questions, including measures of the protein half-life and elimination rate, renal toxicity, GFP immune-related issues, and these would have to be properly addressed. However, our results indicate that the possibility of keeping GFP in vaccines is feasible, and should be considered as a pragmatic way to reduce costs and foster vaccine R\&D.

Acknowledgments We are grateful to Prof. Daniele da Glória de Souza and their team for their help in evaluating the data and for critical advice. MA Rachid and EF Barbosa-Stancioli are CNPq Fellowship Recipients.

Funding information This research was supported by CAPES and the Post-Graduation program in Microbiology from the Universidade Federal de Minas Gerais. Financial resources came also from grants by FAPEMIG and CNPq.

\section{Compliance with ethical standards}

All procedures reported here are in accordance with the ethical principles of animal experimentation adopted by the Ethics Committee for Animal experiments from Universidade Federal de Minas Gerais (CETEA/ UFMG-protocol 273/2008).

Conflict of interest The authors declare that they have no conflict of interest.

\section{References}

1. Luyten J, Beutels P (2016) The social value of vaccination programs: beyond cost-effectiveness. Health Aff (Millwood) 35:212218

2. Buckland BC (2005) The process development challenge for a new vaccine. Nat Med 11:16-19

3. Plotkin SA, Mahmoud AAF, Farrar J (2015) Establishing a global vaccine-development fund. N Engl J Med 373:297-300

4. Andrade LM, Cox L, Versiani AF, da Fonseca FG (2017) A growing world of small things: a brief review on the nanostructured vaccines. Futur Virol 12:767-779

5. Moss B (2007) Poxviridae: the viruses and their replication. In: Fields Virology 2, 5th edn. Lippincott Williams \& Wilkins, Philadelphia, pp 2637-2267

6. Moss B (2011) Smallpox vaccines: targets of protective immunity. Immunol Rev 239:8-26

7. Quinan BR, Daian e Silva DSO, Coelho FM, Da Fonseca FG (2014) Modified vaccinia virus Ankara as vaccine vectors in human and veterinary medicine. Futur Virol 9:173-187

8. Drexler I, Staib C, Sutter G (2004) Modified vaccinia virus Ankara as antigen delivery system: how can we best use its potential? Curr Opin Biotechnol 15:506-512

9. Moss B (2013) Reflections on the early development of poxvirus vectors. Vaccine. 31:4220-4222

10. Zuverink M, Barbieri JT (2015) From GFP to $\beta$-lactamase: advancing intact cell imaging for toxins and effectors. Pathog Dis 73: ftv097

11. Bisht H, Roberts A, Vogel L, Bukreyev A, Collins PL, Murphy BR, Subbarao K, Moss B (2004) Severe acute respiratory syndrome coronavirus spike protein expressed by attenuated vaccinia virus protectively immunizes mice. Proc Natl Acad Sci U S A 101: 6641-6646

12. Souza DG, Cara DC, Cassali GD, Coutinho SF, Silveira MR, Andrade SP, Poole SP, Teixeira MM (2000) Effects of the PAF receptor antagonist UK74505 on local and remote reperfusion injuries following ischaemia of the superior mesenteric artery in the rat. Br J Pharmacol 131:1800-1808

13. Barcelos LS, Talvani A, Teixeira AS, Cassali GD, Andrade SP, Teixeira MM (2004) Production and in vivo effects of chemokines CXCL1-3/KC and CCL2/JE in a model of inflammatory angiogenesis in mice. Inflamm Res 53:576-584

14. Ogawa H, Inouye S, Tsuji FI, Yasuda K, Umesono K (1995) Localization, trafficking, and temperature-dependence of the Aequorea green fluorescent protein in cultured vertebrate cells. Proc Natl Acad Sci U S A 92:11899-11903

15. Ramirez JC, Finke D, Esteban M, Kraehenbuhl JP, Acha-Orbea H (2003) Tissue distribution of the Ankara strain of vaccinia virus (MVA) after mucosal or systemic administration. Arch Virol 148: $827-839$

16. van der Veen BS, de Winther MP, Heeringa P (2009) Myeloperoxidase: molecular mechanisms of action and their relevance to human health and disease. Antioxid Redox Signal 11: 2899-2937

17. Bekheet IW, Madkour ME, Ghaffar NA, Nosseir MMF, Moussa MM, Ibraheim RA, Ateya ME (2009) The role of myeloperoxidase in hepatitis $\mathrm{C}$ virus infection and associated liver cirrhosis. Open Trop Med J 2:1-7

18. Kothari N, Keshari RS, Bogra J, Kohli M, Abbas H, Malik A, Dikshit M, Barthwal MK (2011) Increased myeloperoxidase enzyme activity in plasma is an indicator of inflammation and onset of sepsis. J Crit Care 26:435-e1-435-e.7

19. Prokopowicz Z, Marcinkiewicz J, Katz DR, Chain BM (2012) Neutrophil myeloperoxidase: soldier and statesman. Arch Immunol Ther Exp 60:43-54 
20. Klebanoff SJ (2005) Myeloperoxidase: friend and foe. J Leukoc Biol 77:598-625

21. Edwards L, Wanless IR (2013) Mechanisms of liver involvement in systemic disease. Best Pract Res Clin Gastroenterol 27:471-483

22. Campos F, Rodríguez-Yáñez M, Castellanos M, Arias S, PérezMato M, Sobrino T, Blanco M, Serena J, Castillo J (2011) Blood levels of glutamate oxaloacetate transaminase are more strongly associated with good outcome in acute ischaemic stroke than glutamate pyruvate transaminase levels. Clin Sci 121:11-17

23. Ozer J, Ratner M, Shaw M, Bailey W, Schomaker S (2008) The current state of serum biomarkers of hepatotoxicity. Toxicology. 245:194-205

24. Earl PL, Americo JL, Moss B (2017) Insufficient innate immunity contributes to the susceptibility of the castaneous mouse to orthopoxvirus infection. J Virol 91:e01042-e01017

25. Dowall SD, Graham VA, Rayner E, Hunter L, Watson R, Taylor I et al (2016) Protective effects of a Modified Vaccinia Ankara-based vaccine candidate against Crimean-Congo Haemorrhagic Fever vi- rus require both cellular and humoral responses. PLoS One 11(6): e0156637

26. Langenmayer MC, Lülf-Averhoff AT, Adam-Neumair S, Fux R, Sutter G, Volz A (2018) Distribution and absence of generalized lesions in mice following single dose intramuscular inoculation of the vaccine candidate MVA-MERS-S. Biologicals. 54:58-62

27. Domínguez LE, Brandmüller C, Zorn J, Kirschning CJ, Sutter G, Lehmann MH et al (2014) Chemokine (C-C Motif) receptor 1 is required for efficient recruitment of neutrophils during respiratory infection with modified vaccinia virus Ankara. J Virol 88:10840 10850

28. Price PJ, Bánki Z, Scheideler A, Stoiber H, Verschoor A, Sutter G et al (2015) Complement component C5 recruits neutrophils in the absence of $\mathrm{C} 3$ during respiratory infection with modified vaccinia virus Ankara. J Immunol 194:1164-1168

Publisher's note Springer Nature remains neutral with regard to jurisdictional claims in published maps and institutional affiliations. 